\title{
Towards active photonic dispersion control using graphene-induced non-radiative loss
}

\author{
Jérémy Lhuillier, Pierre Demongodin, Thomas Wood, Malik Kemiche, Bertrand \\ Vilquin, Geneviève Grenet, Sébastien Cueff, Pedro Rojo-Romeo, Xavier Letartre and \\ Christelle Monat \\ Institut des Nanotechnologies de Lyon, Ecole Centrale de Lyon, Université de Lyon, 36 avenue Guy de \\ Collongue, 69134 Ecully cedex, France. \\ jeremy.lhuillier@ec-lyon.fr
}

\begin{abstract}
We show that the photonic dispersion of a two coupled-mode system can be actively tuned using graphene-induced non-radiative loss. Our implementation exploits the spatial modulation of graphene's absorption via patterned oxide substrates. (C) 2020 The Author(s)
\end{abstract}

\section{Introduction}

The optoelectronic properties of graphene make it promising for achieving actively tunable surface-addressable components [1]. In particular, graphene's conductivity highly depends on its chemical potential, thus allowing electrical and optical control using electrical gating and optical pumping respectively. Nonetheless, operation at telecom and shorter wavelengths remains a challenge as graphene mainly provides tunable absorption [2] in this band, while the related limited refractive index change has little effect on the device photonic dispersion [3].

We here develop a model showing that graphene-induced non-radiative losses can have a major effect on the photonic dispersion of a system composed of two coupled modes that interact differently with graphene. While graphene has a limited effect on isolated optical modes, it can strongly affect the coupling between the two modes, hence the photonic dispersion of the system. We then propose an implementation for achieving practical components exploiting this effect.

\section{Graphene-induced loss effect on mode coupling}

Considering two coupled modes at frequencies $\omega_{1}$ and $\omega_{2}$ with the coupling strength $\kappa$ and the associated nonradiative loss rates $\eta_{1}$ and $\eta_{2}$ respectively, frequencies of the resulting coupled-modes are given by:

$$
\Omega_{ \pm}=\frac{\omega_{1}+\omega_{2}}{2}-i \frac{\eta_{1}+\eta_{2}}{2} \pm \sqrt{\left(\frac{\omega_{1}-\omega_{2}}{2}-i \frac{\eta_{1}-\eta_{2}}{2}\right)^{2}+\kappa^{2}}
$$

Unlike what would occur for either mode separately interacting with graphene, equation (1) shows that the graphene induced non-radiative loss rates do contribute to the real part of the coupled-mode frequencies. In particular, when $\omega_{1}=\omega_{2}$, figure 1 shows that the difference in the two mode non-radiative loss rates tends to decrease the coupling between the modes, hence closing the gap that was opened by the mode coupling.
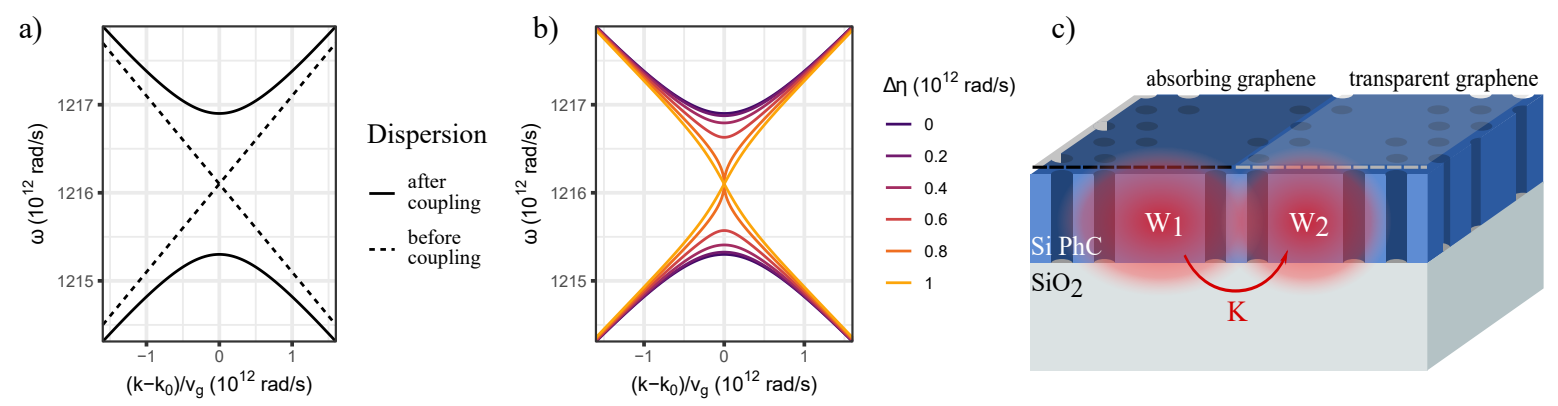

Fig. 1. Effect of coupling and graphene-induced loss on two modes with linear dispersion and opposite group velocities: a) coupled-mode dispersion without loss and b) effect of graphene-induced loss with $\Delta \eta=\left(\eta_{1}-\eta_{2}\right) / 2$ and c) implementation with two coupled photonic crystal waveguides. 
Introduction of non-radiative loss with different rates on two primary modes then allows to reduce the frequency difference of the resulting coupled-modes, thus allowing to modify the photonic dispersion by acting on nonradiative loss in the system. Hence by electrically or optically tuning graphene's chemical potential, dispersion can further be actively controlled.

The monolayer nature of graphene provides a natural way to induce unbalanced loss on two modes by using whether its atomic thickness or its anisotropic permittivity. Furthermore, graphene induced loss can be distributed unequally between two modes according to their field distribution by patterning graphene or by controlling its chemical potential over space. We here explore the latter by exploiting charge transfer between graphene and high work-function oxides.

\section{Spatial modulation of graphene's chemical potential}

When transferred onto a high work-function oxide, graphene's chemical potential is expected to be shifted towards lower energies [4]. By patterning the oxide layer, graphene's chemical potential can then be modulated spatially. We here investigate such phenomena by transferring graphene on a patterned 10nm-thick layer of tungsten oxide $\left(\mathrm{WO}_{3}\right)$ fabricated by sputtering and lift-off on a $90 \mathrm{~nm}$-thick silica layer on silicon substrate. Interaction between $\mathrm{WO}_{3}$ and graphene is first probed by Raman spectroscopy. By comparing with graphene on silica, we show that $\mathrm{WO}_{3}$ induces a chemical potential shift of about $0.1 \mathrm{eV}$ towards lower energies [5]. This shift is also confirmed using nano-XPS linescan accross a pattern. Carbon core level $(\mathrm{C} 1 \mathrm{~s})$ peak position of graphene on $\mathrm{WO}_{3}$ indeed shifts by $0.1 \mathrm{eV}$ compared to graphene on silica. Furthermore, tungsten core levels (W4f) suggest that $\mathrm{WO}_{3}$ is not fully oxidized, suggesting the chemical potential shift can further be increased.
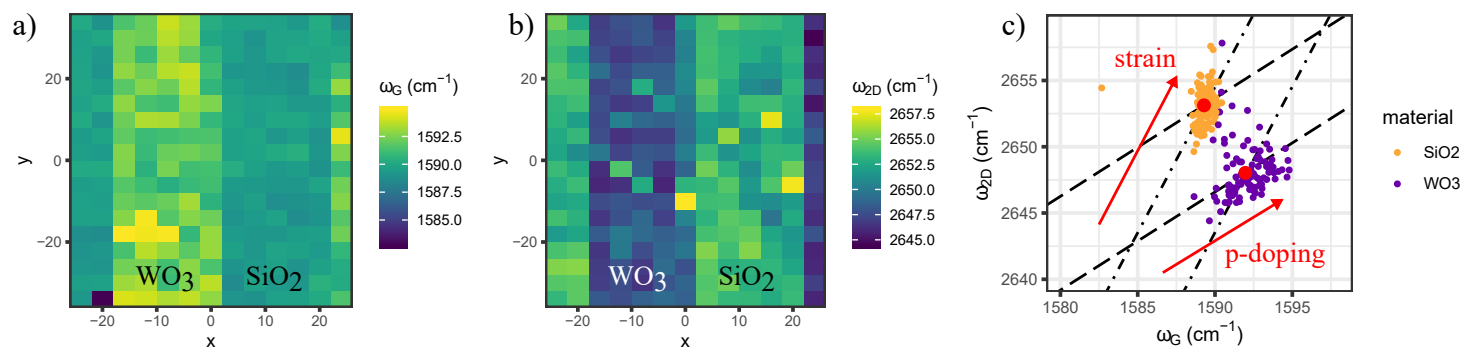

Fig. 2. Raman spectroscopy cartography of graphene on patterned $\mathrm{WO}_{3}$ on $\mathrm{SiO}_{2} / \mathrm{Si}$ substrate showing spatial variation of graphene-related peak frequencies on a) the $\mathrm{G}$ band and b) the 2D band. c) Comparison of the peak frequencies depending on the supporting material allowing to decompose contribution from strain and doping [5].

\section{Conclusion}

We showed that graphene-related non radiative losses on photonic systems composed on two coupled modes can prove useful regarding dispersion engineering and further allow active control of the system optical response. This generic approach can be achieved provided that graphene induces distinct loss rates on the two coupled modes. We proposed and experimentally investigated an implementation that uses patterned high work-function oxide substrates, thus adding possibilities in graphene integration. Beyond active control of component's optical properties, the presented system may provide a canvas to explore non-Hermitian photonics as well as dynamical systems using non-linear properties of graphene.

This work is funded by the European Research Council H2020 Consolidator Grant GRAPHICS $\left(\mathrm{n}^{\circ} 648546\right)$.

\section{References}

1. C. U. Hail et al., "Optical Metasurfaces: Evolving from Passive to Adaptive," Adv. Opt. Mater. 1801786, 1801786 (2019).

2. M.-S. Hwang et al., "Switching of Photonic Crystal Lasers by Graphene," Nano Lett. 17, 1892-1898 (2017).

3. B. Yao et al., "Gate-tunable frequency combs in graphene-nitride microresonators," Nature 558, 410-414 (2018).

4. J. Meyer et al., "Metal oxide induced charge transfer doping and band alignment of graphene electrodes for efficient organic light emitting diodes," Sci. Reports 4, 1-7 (2014).

5. J. E. Lee et al., "Optical separation of mechanical strain from charge doping in graphene," Nat. Commun. 3, 1024 (2012). 